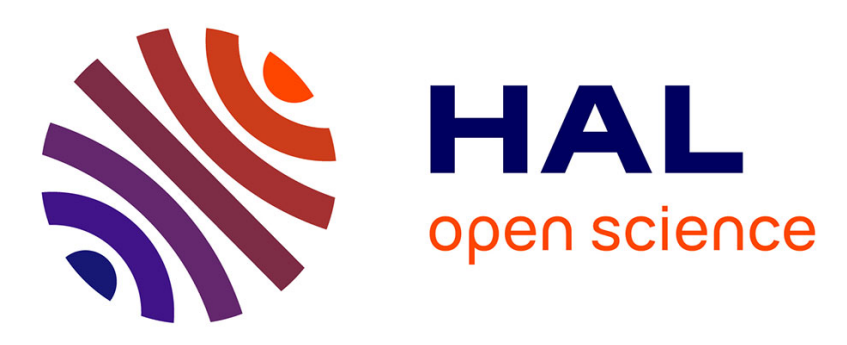

\title{
Linear Adaptive Computed Torque Control for Singularity Crossing of Parallel Robots
}

Adrien Koessler, Nicolas Bouton, Sébastien Briot, Belhassen-Chedli

Bouzgarrou, Youcef Mezouar

\section{To cite this version:}

Adrien Koessler, Nicolas Bouton, Sébastien Briot, Belhassen-Chedli Bouzgarrou, Youcef Mezouar. Linear Adaptive Computed Torque Control for Singularity Crossing of Parallel Robots. ROMANSY 22 - Robot Design, Dynamics and Control, Jun 2018, Rennes, France. pp.222-229, 10.1007/978-3319-78963-7 . hal-02099955

\section{HAL Id: hal-02099955 \\ https://hal.science/hal-02099955}

Submitted on 24 Jun 2019

HAL is a multi-disciplinary open access archive for the deposit and dissemination of scientific research documents, whether they are published or not. The documents may come from teaching and research institutions in France or abroad, or from public or private research centers.
L'archive ouverte pluridisciplinaire HAL, est destinée au dépôt et à la diffusion de documents scientifiques de niveau recherche, publiés ou non, émanant des établissements d'enseignement et de recherche français ou étrangers, des laboratoires publics ou privés. 


\title{
Linear Adaptive Computed Torque Control for Singularity Crossing of Parallel Robots
}

\author{
A. Koessler ${ }^{\dagger}$, N. Bouton ${ }^{\dagger}$, S. Briot ${ }^{*}$, B. C. Bouzgarrou ${ }^{\dagger}$, and Y. Mezouar ${ }^{\dagger}$ \\ $\dagger$ Université Clermont Auvergne, CNRS, SIGMA, Institut Pascal, France \\ * Laboratoire des Sciences du Numérique de Nantes (LS2N), CNRS, France
}

\begin{abstract}
The operational workspace of parallel robots is often reduced by the presence of singularities. Recently, it has been proven that Type 2 singularities can be crossed in a way such that the dynamic model of the robot never degenerates. This discovery has been the starting point of several works on multi-model Computed Torque Control (CTC) that allow crossing of Type 2 singularities. In this paper, we propose a further improvement thanks to adaptive control. The major contribution of the paper is in the control law synthesis, which uses only linear methods, in contrast to usual approaches based on Lyapunov theory. This theoretical development will be validated both in simulation and experimentally.
\end{abstract}

\section{Introduction}

It is often said that parallel manipulators have the edge on their serial counterparts in terms of cycle time and rigidity. However, the size of their workspace is a serious drawback. In general, this workspace is further split by Type 2 singularities (Gosselin and Angeles, 1990). This is especially annoying as joint control usually computes divergent setpoints in the neighborhood of those singularities, meaning that crossing them is not a solution.

A lot of propositions revolve around the idea of getting rid off singularities. Optimal design allows to eliminate them or to reduce their impact (Gogu, 2004). Actuation redundancy or variable actuation modes (Rakotomanga et al., 2008) allows to cross them at a supplementary cost.

Contrary to these approaches, some researchers have proved that Type 2 singularities do not always induce the degeneracy of the inverse dynamical model of parallel robots (Ider, 2005), (Briot and Arakelian, 2008). The expression of these non-degeneracy conditions and their use in multi-model control law will be explained further on.

While being fairly reliable, this method does not ensure success in singularity crossing, mainly because of trajectory tracking error induced by the 
control law. Then, in some cases, the desired trajectory is not respected and the robot is unable to cross the singularity. Therefore, more advanced control is needed in order to ensure a perfect crossing of the singularity.

Adaptive control is widely used when robot dynamic models are not perfectly known or change over time (Zhang and Wei, 2017). Our application falls into the first case, as the dynamic model has poor precision and crossing trajectories need high accelerations. To simplify implementation and tuning, we propose a control law derived from linear algebra, contrary to usual Lyapunov-based approaches.

The paper is organized as follows: previous work on singularity crossing is recalled in section 2. Main contribution lies in section 3, where the linear adaptive control law is presented. Its efficiency is proved in section 4, with simulations and experiments carried out on a planar parallel robot.

\section{Previous Work on Singularity Crossing}

\subsection{Dynamical Non-Degeneracy Rule}

It has been proven by Ider (2005) and Briot and Arakelian (2008) that parallel singularities do not always induce degeneracy of parallel robot's dynamic model. The rule for non-degenerating motion planing is the following: in singular configurations, the wrench exerted on the end-effector (by inertia and external forces) must be orthogonal to the direction of the uncontrollable motion.

This result can be used to generate a crossing trajectory that ensures the non-degeneracy of the dynamic model as explained in (Pagis et al., 2015).

\subsection{Control Scheme for Singularity Crossing}

Multi-model Computed Torque Control law is recalled as explained in (Pagis et al., 2015).

The control error to minimize is $\mathbf{e}=\mathbf{q}_{d}-\mathbf{q}$ where $\mathbf{q}_{d}$ is the desired joint value and $\mathbf{q}$ the measured joint value. Parallel robot IDM can be generally written as :

$$
\tau=\mathbf{M} \ddot{\mathbf{q}}+\mathbf{h}=\mathbf{M v}+\mathbf{h}
$$

with the mass matrix of the robot $\mathbf{M}(\mathbf{q})$ and the vector of centrifugal effects $\mathbf{h}(\mathbf{q}, \dot{\mathbf{q}})$. The intermediate control input $\mathbf{v}$ allows to perform input-output linearization and is defined by 3rd-order dynamics :

$$
\mathbf{v}=\ddot{\mathbf{q}}_{d}+\mathbf{K}_{v} \dot{\mathbf{e}}+\mathbf{K}_{p} \mathbf{e}+\mathbf{K}_{i} \int \mathbf{e}
$$

where gain matrices $\mathbf{K}_{v}, \mathbf{K}_{p}, \mathbf{K}_{i}$ impose the dynamics of articular error. 
However, in singular configurations, IDM (1) cannot be computed. Yet, as explained in (Pagis et al., 2015), the IDM in singularity has a new nondegenerating expression. Transition between the two models can be achieved using a logistic function $\sigma$. Consequently, a multi-model control scheme was implemented in (Pagis et al., 2015) with the following dynamic models:

- Model 1 - complete model $(\sigma=0)$

$$
\boldsymbol{\tau}=\mathbf{M} \ddot{\mathbf{q}}+\mathbf{h}
$$

- Model 2 - non-degenerating model around singularities $(\sigma=1)$

$$
\boldsymbol{\tau}=\mathbf{M}^{\prime} \ddot{\mathbf{q}}+\mathbf{h}^{\prime}
$$

\section{Linear Synthesis of Adaptive Control Law}

To improve tracking performances, a more advanced control algorithm scheme is required. Adaptive control of robotic manipulators is well-known and has been the subject of many works, recently reviewed in (Zhang and Wei, 2017). Adaptation of dynamic parameters is needed to reduce modeling error, resulting in a better trajectory tracking. This could be especially useful when using the less precise non-degenerating model around the singularities. In this paper, contrary to usual approaches, the presented law for parameter adaptation is based on linear techniques.

\subsection{Modeling Error}

This section presents the usual computation of the error between the $\left(n_{d} \times 1\right)$ vector of real dynamic parameters $\chi$ and its estimate $\hat{\boldsymbol{\chi}}$.

The real dynamic model of the robot and the one used for control are

$$
\boldsymbol{\tau}=\mathbf{M}(\chi) \ddot{\mathbf{q}}+\mathbf{h}(\chi) \quad \text { and } \quad \boldsymbol{\tau}=\hat{\mathbf{M}}(\hat{\chi}) \mathbf{v}+\hat{\mathbf{h}}(\hat{\chi})
$$

with $\mathbf{v}$ defined in (2). Modifying (5) allows to get the classical result :

$$
\ddot{\mathbf{e}}+\mathbf{K}_{v} \dot{\mathbf{e}}+\mathbf{K}_{p} \mathbf{e}+\mathbf{K}_{i} \int \mathbf{e}=\hat{\mathbf{M}}^{-1}((\mathbf{M}-\hat{\mathbf{M}}) \ddot{\mathbf{q}}+\mathbf{h}-\hat{\mathbf{h}}) .
$$

Full computation of (6) can be found in (Khalil and Dombre, 2001). Since the dynamic model is linear with regards to parameters $\chi$, one can write

$$
\boldsymbol{\tau}=\mathbf{\Phi} \chi=\mathbf{M} \ddot{\mathbf{q}}+\mathbf{h}
$$

where $\boldsymbol{\Phi}$ is a $\left(n \times n_{d}\right)$ matrix. Consequently, the differential equation on articular error is linked to dynamic parameter error, that is the difference $\tilde{\chi}=\chi-\hat{\chi}$ between real and estimated value:

$$
\ddot{\mathbf{e}}+\mathbf{K}_{v} \dot{\mathbf{e}}+\mathbf{K}_{p} \mathbf{e}+\mathbf{K}_{i} \int \mathbf{e}=\hat{\mathbf{M}}^{-1} \mathbf{\Phi} \tilde{\boldsymbol{\chi}} .
$$




\subsection{Adaptation Law based on State Feedback}

Equation (8) can be put under the form of a state-space model, where state vector $\mathbf{X}$ is a vector of articular error, its integral and its derivative:

$$
\mathbf{X}=\left(\begin{array}{c}
\int \mathbf{e} \\
\mathbf{e} \\
\dot{\mathbf{e}}
\end{array}\right) \Rightarrow \dot{\mathbf{X}}=\left(\begin{array}{ccc}
\mathbf{0}_{n} & \mathbf{1}_{n} & \mathbf{0}_{n} \\
\mathbf{0}_{n} & \mathbf{0}_{n} & \mathbf{1}_{n} \\
-\mathbf{K}_{i} & -\mathbf{K}_{p} & -\mathbf{K}_{v}
\end{array}\right) \mathbf{X}+\left(\begin{array}{c}
\mathbf{0}_{n} \\
\mathbf{0}_{n} \\
\mathbf{1}_{n}
\end{array}\right) \hat{\mathbf{M}}^{-1} \mathbf{\Phi} \tilde{\boldsymbol{\chi}}
$$

The state-space model (9) can be written as:

$$
\dot{\mathbf{X}}=\mathbf{A X}+\mathbf{B w} \quad \text { with } \quad \mathbf{w}=\hat{\mathbf{M}}^{-1} \mathbf{\Phi} \tilde{\chi}
$$

where state matrix $\mathbf{A}$ and input matrix $\mathbf{B}$ are used to check the controllability of the system using Kalman criterion. Then, instead of using Lyapunov theory (Khalil and Dombre, 2001), we stick to linear control methods and use classical state feedback

$$
\mathbf{w}=-\mathbf{L}_{s} \mathbf{X}
$$

where the gain matrix $\mathbf{L}_{s}$ is computed by using pole placement methods in order to ensure that closed-loop state matrix $\mathbf{F}-\mathbf{G L}_{s}$ is a Hurwitz one. Then, state vector is ensured to exponentially converge towards zero. Finally, the estimated dynamic parameters $\hat{\chi}$ are obtained by imposing a first order dynamic evolution on the estimation error $\tilde{\chi}$ :

$$
\dot{\tilde{\chi}}=-\mathbf{G} \tilde{\chi}, \quad \mathbf{G}>0 .
$$

By combining (10), (11) and (12), one can obtain

$$
\dot{\tilde{\chi}}=-\mathbf{G} \boldsymbol{\Phi}^{-1} \hat{\mathbf{M}} \mathbf{L}_{s} \mathbf{X} .
$$

The stability of the control law (13) is ensured by the linear methods used to synthesize it ((11) and (12)). Finally, dynamic parameters are estimated by numerical integration of (13), resulting in the adaptive control scheme presented in Fig. 1.

\section{Case Study on DexTAR Robot}

\subsection{DexTAR Dynamic Modeling}

To check the efficiency of the proposed approach, experiments are carried on a five-bar parallel robot, the DexTAR manufactured by Mecademic, 


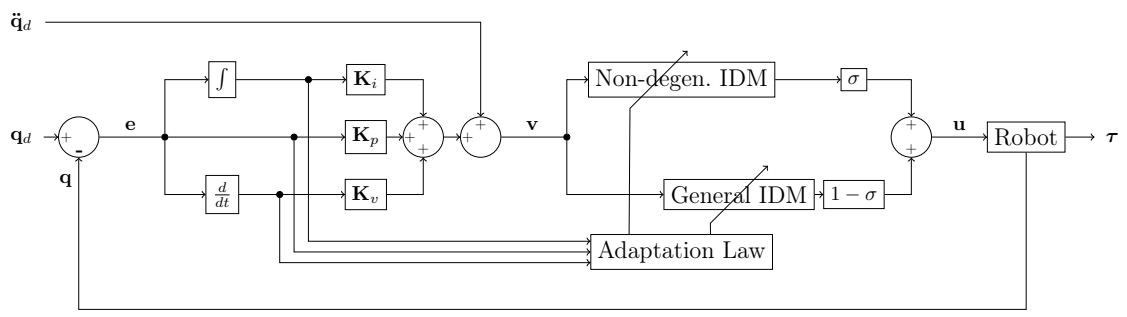

Figure 1: Proposed multi-model adaptive control scheme

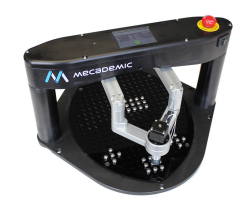

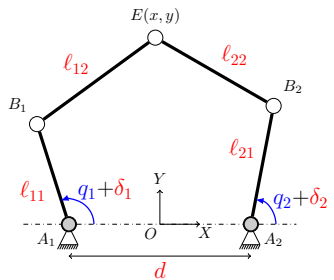

Figure 2: DexTAR geometry

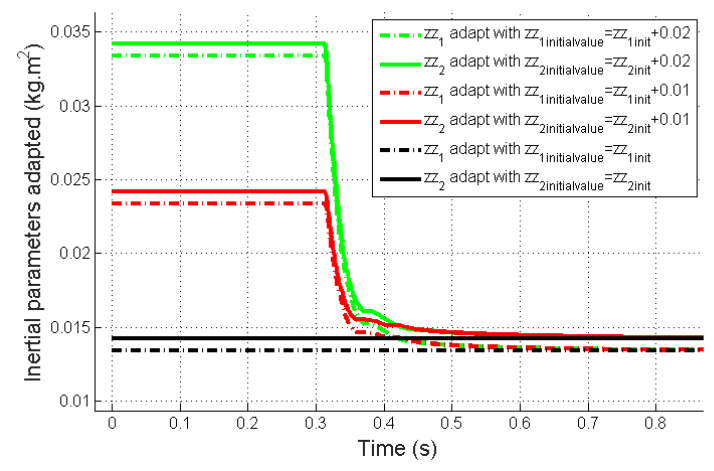

Figure 3: $z z$ adaptation in simulation

which has two degrees of freedom. Its geometry is presented on Fig. 2. The end-effector of the robot is $E$, which vector of Cartesian coordinates is $\mathbf{x}$. The robot is in a Type 2 singularity when distal elements $B_{1} E$ and $B_{2} E$ are aligned.

The inverse dynamic model used in the multi-model control law (see (Pagis et al., 2015)) is :

$$
\boldsymbol{\tau}=\mathbf{Z Z} \ddot{\mathbf{q}}+\mathbf{F}_{s} \operatorname{sign}(\dot{\mathbf{q}})+\mathbf{f}_{f}+(1-\sigma) \mathbf{J}^{T} m \ddot{\mathbf{x}}
$$

It is well known that parameters to adapt have to be chosen carefully. The choice can be further helped by a sensitivity analysis (see (Pagis, 2015)). Based on this analysis, the two parameters $z z_{11}$ and $z z_{21}$ of the matrix $\mathbf{Z Z}$ (see (14)) are adapted. They are expected to have a strong impact on torque setpoint computation, since high articular accelerations are needed to cross the singularities. 


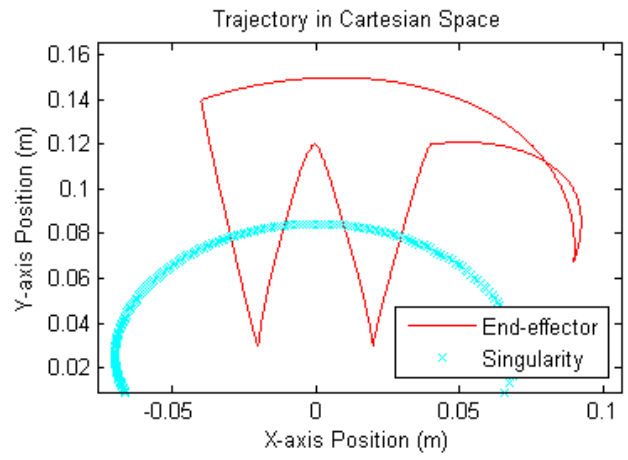

Figure 4: Testing trajectory

\subsection{Simulation Results}

In order to verify that the proposed adaptive control scheme works as intended, wrong initial values of $z z_{11}$ and $z z_{21}$ are fed to the algorithm. Simulations on Matlab/Simulink have been carried out on a standard trajectory that does not cross singularities. As a result, it can be seen in Fig. 3 that estimates of inertia converge back to the real values. This means that the proposed control law is stable and robust. Moreover, articular error is drastically reduced by the proposed control law.

\subsection{Experimental Results}

Now that theoretical stability and performance are verified on simulation, we want to see if adaptive control can help singularity crossing. For this purpose, a trajectory where the singularity is crossed four times was generated, obviously abiding to the non-degeneracy rule (see Fig. 4).

The difficulty induced by singularity crossing is that the robot has to find a configuration where distal legs are fully extended in order to cross the singularity. Articular errors can either lead to crossing failure (not extending enough) or peaks in torque setpoint (trying to extend more than robot geometry allows to).

The adaptive scheme proposed will be judged on several criteria: articular error, setpoint torque values and setpoint torque smoothness.

Experiments are run with unbiased initial values, which correspond to the identified ones $\left(\hat{z} z_{11}=0.0134\right.$ and $\left.\hat{z} z_{21}=0.0142 \mathrm{kgm}^{2}\right)$. Afterwards, initial values are changed by adding or subtracting $0.01 \mathrm{kgm}^{2}$. Logically, introducing biased parameters is expected to favor adaptive control. The improvement obtained with adaptive control is summarized in Table 1. 


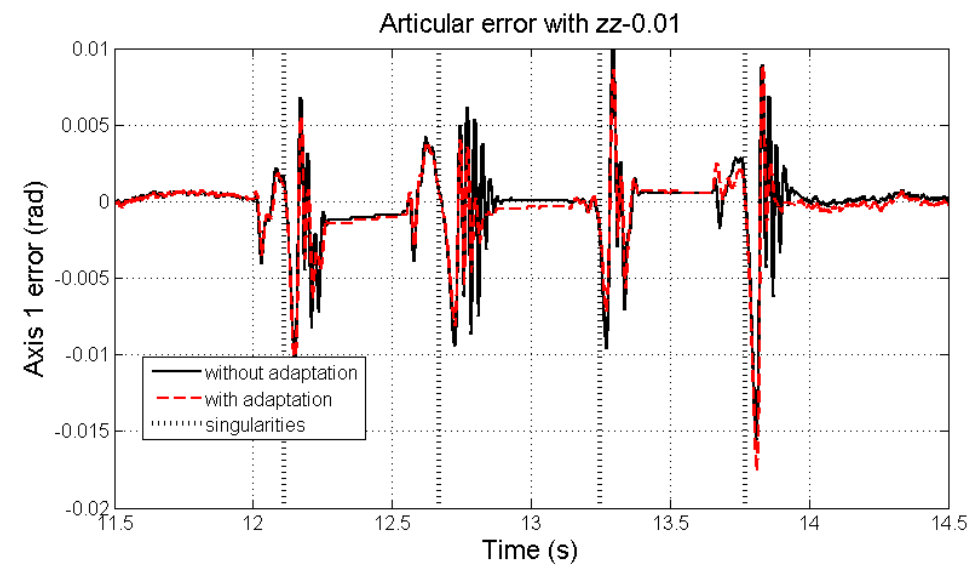

Figure 5: Articular error comparison

Table 1: Experimental results

\begin{tabular}{|c|cc|cc|cc|}
\hline data & \multicolumn{2}{|c|}{$\hat{z z}=z z$} & \multicolumn{2}{c|}{$\hat{z z}=z z+0.01$} & \multicolumn{2}{c|}{$\hat{z}=z z-0.01$} \\
\hline & no adapt & adapt & no adapt & adapt & no adapt & adapt \\
\hline RMS error $(\mathrm{mrad})$ & 2.82 & 3.70 & 3.26 & 3.07 & 14.20 & 5.53 \\
peak error $(\mathrm{mrad})$ & 9.03 & 10.63 & 10.24 & 8.91 & 46.99 & 17.05 \\
RMS torque $(\mathrm{V})$ & 1.37 & 0.96 & 2.26 & 1.71 & 1.61 & 0.89 \\
peak torque $(\mathrm{V})$ & 8 & 3.18 & 8 & 8 & 5.87 & 3.18 \\
peak t. derivative $(\mathrm{V} / \mathrm{s})$ & 1680 & 715 & 2209 & 1949 & 1079 & 302 \\
\hline
\end{tabular}

It can be seen that in all cases, adaptive control reduces input torque peaks and improves smoothness, which is our first goal. Articular error is reduced only if initial parameters are biased. With initial parameters set as $z z-0.01$, error reduction is particularly impressive, as witnessed on Fig 5.

Overall, the proposed control scheme works as intended and is a good alternative for smoothing of torque setpoints, even when the initial values of parameters are precise. Articular error is kept low before reaching the singularity, which is crucial for the success of the crossing.

\section{Conclusion}

Crossing singularities is a potential improvement for the use of parallel robots. It allows to increase the size of operational workspace, which is one of their main downsides. An approach based only on optimal trajectory generation and dedicated control was proposed to achieve singularity cross- 
ing. For the crossing to succeed, it is crucial that the trajectory is precisely tracked around singularities. To do so, an advanced control solution was developed using adaptive control algorithm. The originality of our work is the synthesis of the adaptation law based solely on linear methods.

The proposed control law proved to be stable and robust. It was shown experimentally that it is beneficial in terms of trajectory tracking, torque values and smoothness. Future work consists in transposing the proposed control scheme to predictive control, in order to improve the estimation of dynamic parameters and expected to yield better anticipation of singularity crossing.

\section{Bibliography}

S. Briot and V. Arakelian. Enlarging parallel robot workspace through type2 singularity crossing. International Journal of Robotics Research, 39: 1-11, 2008.

G. Gogu. Structural synthesis of fully-isotropic translational parallel robots via theory of linear transformations. European Journal of Mechanics A/Solids, 6:1021-1039, 2004.

C. Gosselin and J. Angeles. Singularity analysis of closed-loop kinematic chains. IEEE Transactions on Robotics and Automation, 6:281-290, 1990.

S. Ider. Inverse dynamics of parallel manipulators in the presence of drive singularities. Mechanism and Machine Theory, 40:33-44, 2005.

W. Khalil and E. Dombre. Modeling, Identification and Control of Robots. Elsevier, 2001.

G. Pagis, N. Bouton, S. Briot, and P. Martinet. Optimal force generation in parallel manipulators for passing through the singular positions. Control Engineering Practice, 27:967-983, 2015.

Georges Pagis. Augmentation de la taille de l'espace de travail opérationnel des robots parallèles en traversant les singularités de Type 2 : génération de trajectoires optimales et commande avancée. PhD thesis, Ecole Centrale de Nantes, 2015.

N. Rakotomanga, D. Chablat, and S. Caro. Kinetostatic performance of a planar parallel mechanism with variable actuation. In P. Wenger J. Lenarcic, editor, Advances in Robot Kinematics: Analysis and Design. Springer, 2008.

D. Zhang and B. Wei. A review on model reference adaptive control of robotic manipulators. In F. Lamnabhi-Lagarrigue, editor, Annual Reviews in Control 43. Elsevier, 2017. 